\title{
Patient-Reported Outcomes in Onychomycosis: A Review of Psychometrically Evaluated Instruments in Assessing Treatment Effectiveness
}

\author{
Jenny Wang ${ }^{a}$ Lauren E. Wiznia ${ }^{b}$ Evan A. Rieder ${ }^{b}$ \\ ${ }^{a}$ New York University School of Medicine and ${ }^{b}$ The Ronald O. Perelman Department of Dermatology, New York \\ University School of Medicine, New York, NY, USA
}

\section{Keywords}

Nail diseases · Nails · Onychomycosis · Patient-reported outcomes · Psychometrics · Quality of life · Questionnaires

\section{Abstract}

Purpose: Onychomycosis is the most common nail disorder and causes morbidity and impaired quality of life (QOL). Patient-reported outcomes (PRO) are patients' assessment of their health status or treatment response. PROs help assess what is most bothersome to patients to identify targets for intervention. We sought to review the PRO instruments currently used to assess QOL and treatment response in onychomycosis patients. Procedures: A systematic review was performed by searching PubMed, Embase, CINAHL, and PsycINFO databases through December 31, 2016, to identify all English language literature on onychomycosis, PRO, and QOL. Results: Currently, 5 validated PRO instruments exist specifically for onychomycosis. Oral therapies were most extensively studied using PRO instruments. QOL data generally correlated with clinical change, although patients sometimes reported improvement without any clinically significant nail clearance. The only psychometrically validated PRO instrument used to evaluate treatment response is the OnyCOE- $\mathrm{t}^{\mathrm{TM}}$. Conclusions: Clinicians may underestimate the impact of onychomycosis on patients. With recent initiatives from health-care management organizations to improve pa-
\end{abstract}

\section{KARGER}

(C) 2017 S. Karger AG, Basel

E-Mail karger@karger.com

www.karger.com/sad tient experience and the recent approval of expensive and nonsuperior topical antifungal medications, PROs will be increasingly important in onychomycosis to assess patient priorities and optimize treatment. Future research should evaluate these instruments in special populations and fingernail disease.

(c) 2017 S. Karger AG, Basel

\section{Introduction}

Onychomycosis is the most frequent nail disorder, accounting for $50-60 \%$ of nail pathology [1], with toenail disease being about 10 times more common than fingernail disease [2]. Onychomycosis affects over $4 \%$ of people in North America and Europe and imposes a substantial economic burden $[1,3]$. As a disease with mostly benign clinical features, the impact of onychomycosis on patients' quality of life (QOL) is often underestimated. Nail abnormalities can lead to pain, discomfort, limited dexterity and mobility in daily activities [4], and onychomycosis may also be complicated by skin and soft tissue infections [5]. Psychosocial consequences such as embarrassment, decreased self-esteem, anxiety, and depression are often more common in onychomycosis patients than physical sequelae $[4,6]$. 
Patient-reported outcomes (PRO) refer to patients' assessment of their health status or impression of a treatment. Such measurements provide quantifiable data regarding disease impact apart from objective clinical measures. PROs can highlight the most bothersome aspects of onychomycosis for patients and identify targets for intervention [7], thus allowing for the customization of care around patients' goals, treatment optimization, and monitoring of disease activity during therapy [8]. PROs are increasingly becoming an outcome parameter in clinical studies as well as in health-care management and reimbursement [8]. General health questionnaires have limited utility as they are unable to reliably identify problems experienced by dermatologic patients [9]. Instead, disease-specific PRO instruments have been created, validated, and used for onychomycosis.

This review will summarize the current tools used to assess QOL and responsiveness to treatment in onychomycosis patients. First, the 5 current psychometrically validated PRO instruments specific for onychomycosis will be reviewed with a brief discussion of nonvalidated or nonspecific metrics. Then, the use of PROs in evaluating onychomycosis treatment will be discussed.

\section{Methods}

We searched PubMed, Embase, CINAHL, and PsycINFO databases for all published, English language, clinical studies using PRO instruments for onychomycosis through December 31, 2016. Search terms included onychomycosis, patient-reported outcomes, and quality of life. All articles using any PRO instrument to evaluate QOL of onychomycosis patients were included, regardless of study design. While some studies evaluated QOL in onychomycosis patients using nonvalidated or nonspecific tools, we emphasized psychometrically evaluated PRO instruments in our discussion. Articles that studied nail or foot pathology in general and failed to separate onychomycosis from other pathology were excluded. Bibliographies were searched for additional studies that met inclusion and exclusion criteria. Of 168 articles, 25 unique studies with an aggregate of 12,821 patients met the inclusion criteria. Quality of evidence was graded on a scale of $1-5$ per the Oxford Center for Evidence-Based Medicine levels of evidence. Because of the poor quality of evidence, we were unable to complete a quantitative review and thus present our findings qualitatively.

\section{Results}

Of 168 results, 25 unique studies met the inclusion criteria. Eight studies describe baseline characteristics of onychomycosis patients using PRO instruments [10-17], while 8 other studies use nonvalidated instruments or in- struments nonspecific to onychomycosis (Table 1) [7, 18-25]. Ten studies used PRO instruments to evaluate onychomycosis treatments (Table 2) [26-35].

\section{PRO Instruments}

Data collected with PRO instruments are not meaningful without extensive psychometric evaluation; the aspects of this validation are summarized in Table 3. To date, 5 onychomycosis-specific PRO instruments have been validated and are described in Table 4. Overall, all 5 instruments fulfill psychometric validation. Earlier questionnaires contained a generic core of general physical and mental health questions $[10,12,15]$, whereas later instruments consist only of disease-specific items [16, 17], which address symptoms, physical functioning, and psychosocial impact particular to onychomycosis. Multiple nonvalidated PRO instruments have been reported, wherein rationale for item selection and psychometric evaluation were absent [18-23].

Lubeck et al. [10,18] produced the first instrument measuring QOL in onychomycosis patients, the Onychomycosis QOL Questionnaire, which was later revised and validated in patients with toenail onychomycosis. In the validation study for the Lubeck instrument [10], patients with clinical improvement demonstrated statistically significant change in most disease-specific scales over time, whereas no significant change was noted in clinically stable patients, reflecting responsiveness of disease-specific measures.

Turner and Testa [15] later created the Onychomycosis Disease-Specific Questionnaire (ODSQ), which derives questions from the Lubeck instrument and has been validated for both toenail and fingernail disease. The OnyCOE- $\mathrm{t}^{\mathrm{TM}}$ instrument is another questionnaire developed and modified from the Lubeck instrument [16]. When comparing OnyCOE- $\mathrm{t}^{\mathrm{TM}}$ scores after treatment with baseline, the clinically improved group of subjects had statistically significant change in all domains including symptom frequency and bothersomeness, physical activities problems, appearance problems, and stigma. However, the clinically unimproved group also had significant changes for 5 out of 7 domains. Uniquely, clinical meaningfulness was evaluated by estimating the minimally clinical important difference (MCID), i.e. how much a scale needs to change to reflect a corresponding clinical change. The authors found that an 8.5-point change on their 100-point scale corresponded to a $25 \%$ improvement in nail clearance. The MCID allows physicians to correlate clinical findings with PRO data. Weaknesses include lack of variability or test-retest evaluation

Onycho PRO 
Table 1. Summary of PRO instruments for onychomycosis QOL evaluation

\begin{tabular}{|c|c|c|c|c|c|}
\hline $\begin{array}{l}\text { First author } \\
\text { [Ref.], year }\end{array}$ & $\begin{array}{l}\text { Study design } \\
\text { (patients, } \mathrm{n} \text { ) }\end{array}$ & PRO Instrument (questions, n) & PRO outcomes & Limitations & QOE \\
\hline $\begin{array}{l}\text { Lubeck [18], } \\
1993 \\
\text { Lubeck [19], } \\
1998\end{array}$ & $\begin{array}{l}\text { Case control, } \\
\text { cross-sectional } \\
(299)\end{array}$ & $\begin{array}{l}\text { Nonvalidated, OM-specific: validated } \\
\text { generic core (National Health Interview } \\
\text { Survey, Mental Health Index from the } \\
\text { MOS, Fleming Self-Esteem Scales) and } \\
\text { nonvalidated disease-specific items (55) }\end{array}$ & $\begin{array}{l}\text { Generic health questions were less sensitive than disease- } \\
\text { specific questions } \\
\text { Concerns: physical appearance (time/pain involved with } \\
\text { nail care, embarrassment, self-consciousness, concern } \\
\text { with concealing nails), disease symptoms (pain, } \\
\text { thickening, discoloration, deformity), and functional } \\
\text { limitations associated with standing on one's feet or } \\
\text { working with one's fingers } \\
\text { Factors NOT affecting QOL: disease site } \\
\text { Treatment: } 79 \% \text { asked providers about treatment, but } 26 \% \\
\text { said providers discouraged them from taking medication; } \\
88 \% \text { were willing to take a drug with short-term side } \\
\text { effects, } 41 \% \text { would take such a drug even with the risk of } \\
\text { recurrence; } 13 \% \text { were willing to take a drug with long- } \\
\text { term side effects }\end{array}$ & $\begin{array}{l}\text { Nonvalidated PRO } \\
\text { instrument, control group } \\
\text { dissimilar in terms of gender } \\
\text { and age, recall bias, selection } \\
\text { bias }\end{array}$ & 4 \\
\hline $\begin{array}{l}\text { Whittam [20], } \\
1997\end{array}$ & $\begin{array}{l}\text { Case control, } \\
\text { cross-sectional } \\
(60)\end{array}$ & $\begin{array}{l}\text { Nonvalidated, OM-specific: questions } \\
\text { based on responses of a pilot group of } \\
20 \text { patients with OM (9) }\end{array}$ & $\begin{array}{l}\text { Main concerns: nail care, pain, footwear limitations } \\
\text { Economic burden: OM patients spent more money on nail } \\
\text { care and made more visits to clinicians }\end{array}$ & $\begin{array}{l}\text { Nonvalidated PRO } \\
\text { instrument, small sample } \\
\text { size, confounded by subjects } \\
\text { without OM, recall bias, } \\
\text { selection bias }\end{array}$ & 4 \\
\hline $\begin{array}{l}\text { Schein [21], } \\
1997\end{array}$ & $\begin{array}{l}\text { Case series } \\
(150)\end{array}$ & $\begin{array}{l}\text { Nonvalidated, OM-specific: generic core } \\
\text { (physical functioning, social } \\
\text { functioning, pain, health distress, } \\
\text { stigma) and disease-specific (symptoms, } \\
\text { physical activities) questions (NR) }\end{array}$ & $\begin{array}{l}\text { Main concerns: embarrassment (67\%), discomfort (54\%), } \\
\text { footwear limitations ( } 40 \%) \text {, pain ( } 36 \%) \\
\text { Factors worsening QOL: female gender } \\
\text { Provider differences: dermatologists were more likely to } \\
\text { order diagnostic tests and prescribe oral antifungals than } \\
\text { podiatrists; podiatrists were more likely to debride }\end{array}$ & $\begin{array}{l}\text { Nonvalidated PRO } \\
\text { instrument, no mycological } \\
\text { confirmation of OM, small } \\
\text { sample size, no control } \\
\text { group, nonstandardized } \\
\text { treatment, recall bias, } \\
\text { selection bias }\end{array}$ & 4 \\
\hline $\begin{array}{l}\text { Elewski [22], } \\
1997\end{array}$ & $\begin{array}{l}\text { Cross-sectional } \\
(93)\end{array}$ & $\begin{array}{l}\text { Nonvalidated, OM-specific: disease- } \\
\text { specific questions about physical disease } \\
\text { and psychosocial state (15) }\end{array}$ & $\begin{array}{l}92 \% \text { reported negative psychosocial and/or physical effects } \\
\text { Main concerns: nail care }(75 \%) \text {, negative self-image ( } 44 \%) \text {, } \\
\text { pain ( } 41 \%) \text {, transmission to others ( } 31 \%) \text {, feeling } \\
\text { conspicuous ( } 28 \%) \text {, avoiding public facilities ( } 18 \%) \\
\text { Factors worsening QOL: Duration of disease ( } \geq 10 \text { years), } \\
\text { number of nails involved ( } \geq 5 \text { nails) } \\
\text { Treatment: } 66 \% \text { wanted to continue treatment even if } \\
\text { results would be disappointing }\end{array}$ & $\begin{array}{l}\text { Nonvalidated PRO } \\
\text { instrument, small sample } \\
\text { size, no control group, recall } \\
\text { bias, selection bias }\end{array}$ & 4 \\
\hline Drake [23], 1998 & $\begin{array}{l}\text { Cross-sectional } \\
(258)\end{array}$ & $\begin{array}{l}\text { Nonvalidated, OM-specific: disease- } \\
\text { specific questions (patient, physical and } \\
\text { functional, psychosocial, and economic } \\
\text { domains) based on authors' experience, } \\
\text { pilot study, and instrument by Lubeck } \\
\text { et al. [18] (57) }\end{array}$ & $\begin{array}{l}70 \% \text { consider OM at least a moderate problem } \\
\text { Main concerns: nail care }(76 \%) \text {, embarrassment }(75 \%) \text {, } \\
\text { pain }(48 \%) \text {, pressure }(40 \%) \text {, discomfort wearing shoes } \\
(38 \%) \text {, picking up small objects ( } 41 \%) \\
\text { Factors worsening QOL: female gender, fingernail } \\
\text { involvement } \\
\text { Economic burden: in a } 6 \text {-month period, there were } 58 \text { OM- } \\
\text { related sick days and } 468 \text { medical visits ( } 1.8 \text { per subject) } \\
\text { Treatment: } 97 \% \text { stated they would be willing to pay for a } \\
\text { medication with an } 80 \% \text { cure rate, even if their insurance } \\
\text { did not cover the cost; } 57 \% \text { would be willing to pay if cure } \\
\text { rate was } 35 \%\end{array}$ & $\begin{array}{l}\text { Nonvalidated PRO } \\
\text { instrument, no control } \\
\text { group, unclear how subjects } \\
\text { were diagnosed, recall bias, } \\
\text { selection bias }\end{array}$ & 4 \\
\hline Drake [12], 1999 & $\begin{array}{l}\text { Cross-sectional } \\
(532)\end{array}$ & $\begin{array}{l}\text { Validated, OM-specific: generic core } \\
\text { (MOS Short Form Survey, Positive } \\
\text { Well-Being scale from Psychological } \\
\text { General Well-Being Index) and disease- } \\
\text { specific questions (social, emotional, } \\
\text { symptoms) in English, German, French, } \\
\text { and Italian ( } 40 \text { for toenail, } 47 \text { for } \\
\text { fingernail) }\end{array}$ & $\begin{array}{l}\text { Generic health questions were less sensitive than disease- } \\
\text { specific questions } \\
\text { Main concerns: embarrassment, work functioning, } \\
\text { decreased social activity, fear of spread, and pain } \\
\text { Physicians tended to underestimate pain } \\
\text { Factors worsening QOL: female gender, older age, } \\
\text { fingernail involvement, duration of disease, number of } \\
\text { nails involved, proportion of individual nails involved } \\
\text { Cultural differences: German and US patients felt more } \\
\text { seriously affected by OM than Italian patients }\end{array}$ & $\begin{array}{l}\text { No control group, unclear } \\
\text { how subjects were diagnosed, } \\
\text { recall bias, selection bias }\end{array}$ & 4 \\
\hline $\begin{array}{l}\text { Lubeck [10], } \\
1999\end{array}$ & $\begin{array}{l}\text { Case series } \\
(129)\end{array}$ & $\begin{array}{l}\text { Validated, OM-specific: generic core } \\
\text { (Medical Outcomes Study: general } \\
\text { health perceptions, pain, mental health, } \\
\text { social function, health distress, physical } \\
\text { function, health transition) and disease- } \\
\text { specific questions (toe symptom } \\
\text { frequency and bothersomeness, } \\
\text { problems with appearance, problems } \\
\text { with activities, stigma, treatment } \\
\text { satisfaction) (80) }\end{array}$ & $\begin{array}{l}\text { Generic health questions were less sensitive than disease- } \\
\text { specific questions } \\
\text { Factors worsening QOL: female gender }\end{array}$ & $\begin{array}{l}\text { Small sample size, no control } \\
\text { group, no mycological } \\
\text { confirmation of diagnosis, } \\
\text { recall bias, selection bias }\end{array}$ & 4 \\
\hline
\end{tabular}


Table 1 (continued)

\begin{tabular}{|c|c|c|c|c|c|}
\hline $\begin{array}{l}\text { First author } \\
\text { [Ref.], year }\end{array}$ & $\begin{array}{l}\text { Study design } \\
\text { (patients, } \mathrm{n} \text { ) }\end{array}$ & PRO Instrument (questions, n) & PRO outcomes & Limitations & QOE \\
\hline $\begin{array}{l}\text { Lubeck [11], } \\
1999\end{array}$ & $\begin{array}{l}\text { Case series } \\
(113)\end{array}$ & $\begin{array}{l}\text { Validated, OM-specific: questionnaire } \\
\text { developed by Lubeck et al. }[18,19]\end{array}$ & $\begin{array}{l}\text { Provider differences: improvements in QOL were greater } \\
\text { in the group treated by dermatologists, who tended to } \\
\text { prescribe oral antifungals, versus podiatrists, who tended } \\
\text { to debride } \\
\text { Treatment: patients reported improvement in disease- } \\
\text { specific QOL after starting a new treatment, and these } \\
\text { improvements continued over } 9 \text { months of follow-up }\end{array}$ & $\begin{array}{l}\text { Small sample size, no control } \\
\text { group, no mycological } \\
\text { confirmation of diagnosis, } \\
\text { non-standardization of } \\
\text { treatment, recall bias, } \\
\text { selection bias }\end{array}$ & 4 \\
\hline $\begin{array}{l}\text { Turner [15], } \\
2000\end{array}$ & $\begin{array}{l}\text { Double-blind } \\
\text { RCT }(268)\end{array}$ & $\begin{array}{l}\text { Validated, OM-specific: OM Disease- } \\
\text { Specific Questionnaire (ODSQ) with } \\
\text { generic core (MOS: general health and } \\
\text { well-being scales) and disease-specific } \\
\text { questions (symptoms, pain, appearance, } \\
\text { physical activities, social activities, } \\
\text { stigma, treatment satisfaction) (55) }\end{array}$ & $\begin{array}{l}\text { Generic health questions were less sensitive than disease- } \\
\text { specific questions in eliciting QOL differences for clinical } \\
\text { change } \\
\text { Clinical change: cured and improved patients had higher } \\
\text { QOL than the therapeutic failure group, with very large } \\
\text { differences for symptom distress and functional impact } \\
\text { scores; mycologically eradicated patients also had higher } \\
\text { QOL scores than mycologically persistent patients }\end{array}$ & $\begin{array}{l}\text { Poor follow-up with only } \\
268 / 365(73 \%) \text { of enrolled } \\
\text { patients analyzed }\end{array}$ & 1 \\
\hline $\begin{array}{l}\text { Potter [16] } \\
2006\end{array}$ & $\begin{array}{l}\text { Open-label, } \\
\text { RCT (504) }\end{array}$ & $\begin{array}{l}\text { Validated, OM-specific: OnyCOE-t }{ }^{\mathrm{TM}} \\
\text { with disease-specific questions } \\
\text { (symptoms, appearance problems, } \\
\text { physical activities, overall problem, } \\
\text { stigma, treatment satisfaction) (33) }\end{array}$ & $\begin{array}{l}\text { Factors worsening QOL: female gender, young age } \\
\text { Clinical change: mycological cure resulted in higher } \\
\text { scores than uncured patients; an } 8.5 \text {-point change in } \\
\text { OnyCOE- } \mathrm{t}^{\mathrm{TM}} \text { scores correlated with } 25 \% \text { improvement } \\
\text { in target nail clearing }\end{array}$ & $\begin{array}{l}\text { Clinical assessments were } \\
\text { confined to } 1 \text { nail vs. PRO } \\
\text { measures disease on all nails, } \\
\text { differences were minimal as } \\
\text { both groups received } \\
\text { treatment }\end{array}$ & 1 \\
\hline $\begin{array}{l}\text { Warshaw [17], } \\
2007\end{array}$ & $\begin{array}{l}\text { Case Series } \\
(402)\end{array}$ & $\begin{array}{l}\text { Validated, OM-specific: NailQoL with } \\
\text { dermatology-specific questions } \\
\text { (Skindex-29) and disease-specific } \\
\text { questions (emotional, social, symptoms) } \\
\text { (15) }\end{array}$ & $\begin{array}{l}\text { Generic dermatology questions (Skindex-29) were less } \\
\text { sensitive than disease-specific questions in eliciting QOL } \\
\text { differences } \\
\text { Main concerns: self-consciousness, embarrassment, nail } \\
\text { care, concealing nails from others, pain } \\
\text { Factors worsening QOL: fingernail involvement, duration } \\
\text { of disease, number of nails involved } \\
\text { Clinical change: NailQOL scores were improved for } \\
\text { complete cure (clinical and mycological) but not for } \\
\text { mycological cure only } \\
\text { Relative to other diseases (Skindex-29): compared to } \\
\text { eczema and body psoriasis, OM QOL was better; } \\
\text { compared to NMSC and benign growths, OM } \\
\text { symptomatic and functioning impact was similar, but OM } \\
\text { emotional impact is worse }\end{array}$ & $\begin{array}{l}\text { No control group, } \\
\text { confounding due to } \\
\text { terbinafine use, confounding } \\
\text { due to comorbidities, lack of } \\
\text { generalizability (only } \\
\text { veterans included) }\end{array}$ & 4 \\
\hline Tabolli [7], 2007 & $\begin{array}{l}\text { Cross-sectional } \\
\text { study }(66)\end{array}$ & $\begin{array}{l}\text { Validated, dermatology-specific: } \\
\text { Skindex-29 (dermatology-specific), } \\
\text { General Health Questionnaire (generic), } \\
\text { MOS Short Form (generic) (77) }\end{array}$ & $\begin{array}{l}\text { Cultural differences: fingernail disorders impaired QOL } \\
\text { less in Italian patients than other countries } \\
\text { Relative to other diseases (Skindex-29): compared to other } \\
\text { nail disease, OM had worse QOL; compared to dermatitis, } \\
\text { warts, erythema, OM had better QOL } \\
\text { Relative to other disease (MOS): compared to dermatitis, } \\
\text { warts, erythema, OM had better QOL }\end{array}$ & $\begin{array}{l}\text { Use of dermatology PRO } \\
\text { may be less sensitive than } \\
\text { OM-specific PRO, small } \\
\text { sample size, no control } \\
\text { group, recall bias, selection } \\
\text { bias }\end{array}$ & 4 \\
\hline $\begin{array}{l}\text { Szepietowski } \\
{[13], 2007}\end{array}$ & $\begin{array}{l}\text { Cross-sectional } \\
\text { study }(3,904)\end{array}$ & $\begin{array}{l}\text { Validated, OM-specific: questionnaire } \\
\text { developed by Drake et al. [12] in Polish } \\
(40)\end{array}$ & $\begin{array}{l}\text { Main concerns: pain, embarrassment, fear of spread to } \\
\text { other nails, fear of spread to other people, aesthetic } \\
\text { disfigurement } \\
\text { Factors worsening QOL: female gender, fingernail, number } \\
\text { of nails involved ( }>5 \text { ), educational level, towns/cities } \\
\text { residence } \\
\text { Treatment: patients previously unsuccessfully treated had } \\
\text { lower QOL than those who had never undergone therapy }\end{array}$ & $\begin{array}{l}\text { No control group, recall bias, } \\
\text { selection bias }\end{array}$ & 4 \\
\hline $\begin{array}{l}\text { Szepietowski } \\
{[25], 2009}\end{array}$ & $\begin{array}{l}\text { Case series } \\
(1,684)\end{array}$ & $\begin{array}{l}\text { Validated, dermatology-specific: } \\
\text { stigmatization questionnaire (6) }\end{array}$ & $\begin{array}{l}\text { OM patients had increased feeling of stigmatization } \\
\text { Main concerns: others thinking disease is contagious, self- } \\
\text { consciousness, self-image } \\
\text { Factors worsening QOL: female gender, younger age, } \\
\text { fingernail involvement } \\
\text { Factors NOT affecting QOL: duration of disease, number } \\
\text { of nails involved, educational level } \\
\text { Treatment: therapy with combined terbinafine and } \\
\text { amorolfine resulted in reduced stigmatization level to } 40 \% \\
\text { of baseline level }\end{array}$ & $\begin{array}{l}\text { No control group, recall bias, } \\
\text { selection bias }\end{array}$ & 4 \\
\hline
\end{tabular}


Table 1 (continued)

\begin{tabular}{|c|c|c|c|c|c|}
\hline $\begin{array}{l}\text { First author } \\
\text { [Ref.], year }\end{array}$ & $\begin{array}{l}\text { Study design } \\
\text { (patients, n) }\end{array}$ & PRO Instrument (questions, $\mathrm{n}$ ) & PRO outcomes & Limitations & QOE \\
\hline $\begin{array}{l}\text { Milobratovic } \\
{[14], 2013}\end{array}$ & $\begin{array}{l}\text { Cross-sectional } \\
\text { study }(140)\end{array}$ & $\begin{array}{l}\text { Validated, OM-specific: validated } \\
\text { generic core from MOS Short Form } \\
\text { General Health Survey and toenail- } \\
\text { specific scale developed by Drake et al. } \\
\text { [12] validated by authors }(53)\end{array}$ & $\begin{array}{l}\text { Main concerns: nail care, bothersomeness, aesthetic } \\
\text { disfigurement, footwear limitation, fear of spread to other } \\
\text { nails, fear of spread to other people } \\
\text { Factors worsening QOL: female gender, duration of disease } \\
\text { ( } 2-5 \text { years specifically, no difference }>5 \text { years), proportion } \\
\text { of individual nails involved ( } \geq 70 \%) \text {, blue-collar } \\
\text { occupation } \\
\text { Cultural differences: QOL was worse for Serbian patients } \\
\text { than US or Italian patients }\end{array}$ & $\begin{array}{l}\text { Small sample size, no control } \\
\text { group, confounding by } \\
\text { comorbidities, recall bias, } \\
\text { selection bias }\end{array}$ & 4 \\
\hline $\begin{array}{l}\text { Bunyaratavej } \\
{[24], 2015}\end{array}$ & $\begin{array}{l}\text { Cross-sectional } \\
\text { study (110) }\end{array}$ & $\begin{array}{l}\text { Validated, dermatology-specific: DLQI } \\
\text { (symptoms, daily activities, leisure, } \\
\text { personal relationships, treatment) (10) }\end{array}$ & $\begin{array}{l}\text { Factors worsening QOL: female gender, younger age ( }<60 \\
\text { years) } \\
\text { Relative to other disease (DLQI): compared to acne, } \\
\text { pigmentary disorders, and psoriasis, OM QOL is better }\end{array}$ & $\begin{array}{l}\text { Use of dermatology PRO } \\
\text { may be less sensitive than } \\
\text { OM-specific PRO, small } \\
\text { sample size, no control } \\
\text { group, confounding by } \\
\text { different stages of treatment, } \\
\text { recall bias, selection bias }\end{array}$ & 4 \\
\hline
\end{tabular}

DLQI, Dermatology Life Quality Index; MOS, Medical Outcomes Study; NR, not reported; OM, onychomycosis; PRO, patient-reported outcomes; QOL, quality of life; RCT, randomized controlled trial; QOE, quality of evidence.

Table 2. Summary of PRO instruments for onychomycosis treatment evaluation

\begin{tabular}{|c|c|c|c|c|c|c|c|c|}
\hline $\begin{array}{l}\text { Treatment } \\
\text { route, } \\
\text { source }\end{array}$ & $\begin{array}{l}\text { Study } \\
\text { design }\end{array}$ & Comparison & PRO instrument & PRO outcomes & Clinical outcomes & Follow-up & Limitations & QOE \\
\hline $\begin{array}{l}\text { Oral, } \\
\text { Pollak and } \\
\text { Billstein [26] }\end{array}$ & $\begin{array}{l}\text { Case } \\
\text { series } \\
(n= \\
1,534)\end{array}$ & $\begin{array}{l}\text { Terbinafine } \\
\text { ( } 250 \mathrm{mg} \text { daily) } \\
\text { for } 12,18 \text {, and } \\
24 \text { weeks; no } \\
\text { control }\end{array}$ & NR & $\begin{array}{l}\text { More than } 80 \% \text { rated } \\
\text { treatment with terbinafine as } \\
\text { either "very good" or "good"; } \\
68.8 \% \text { were very satisfied or } \\
\text { satisfied with toenail } \\
\text { appearance, } 76.0 \% \text { with } \\
\text { treatment results, and } 76.3 \% \\
\text { with the most bothersome } \\
\text { aspects of the disease, such as } \\
\text { pain with walking or inability } \\
\text { to feel one's toes }\end{array}$ & $\begin{array}{l}\text { Mycological cure rates } \\
\text { were } 72.1 \% \text { ( } 12 \\
\text { weeks), } 72.5 \% \text { ( } 18 \\
\text { weeks), and } 77 \% \text { ( } 24 \\
\text { weeks); clinical cure } \\
\text { rates were } 49.5 \% \text { (12 } \\
\text { weeks), } 49.2 \% \text { ( } 18 \\
\text { weeks), and } 44.6 \% \text { ( } 24 \\
\text { weeks); recurrence } \\
\text { rates ranged from } 14.1 \\
\text { to } 17.3 \%\end{array}$ & $\begin{array}{l}72 \text { weeks, } \\
\text { number lost } \\
\text { to follow-up } \\
\text { NR but } 110 \\
\text { received } \\
\text { insufficient } \\
\text { treatment }\end{array}$ & $\begin{array}{l}\text { No control, no } \\
\text { randomization, } \\
\text { treatment } \\
\text { duration } \\
\text { contingent on } \\
\text { failure to } \\
\text { improve at an } \\
\text { earlier time } \\
\text { point, } \\
\text { questionable } \\
\text { validation of } \\
\text { instrument }\end{array}$ & 4 \\
\hline $\begin{array}{l}\text { Oral, } \\
\text { Stier et al. } \\
{[27]}\end{array}$ & $\begin{array}{l}\text { Case } \\
\text { series } \\
(n=147)\end{array}$ & $\begin{array}{l}\text { Oral therapy } \\
\text { ( } n=58) \text { vs. } \\
\text { non-oral } \\
\text { therapy } \\
\text { (debridement, } \\
\text { topical } \\
\text { medications) } \\
(n=89)\end{array}$ & $\begin{array}{l}\text { Questions from } \\
\text { Lubeck et al. [18] } \\
\text { (nonvalidated, } \\
\text { OM-specific), } \\
\text { Medical Outcomes } \\
\text { Study questionnaire } \\
\text { (validated, generic), } \\
\text { and Short Form of } \\
\text { the Medical } \\
\text { Outcomes } \\
\text { questionnaire } \\
\text { (validated, generic); } \\
\text { however, details of } \\
\text { actual instrument } \\
\text { unclear }\end{array}$ & $\begin{array}{l}\text { Oral therapy group reported } \\
\text { statistically significantly } \\
\text { improved QOL scores }(p< \\
0.05) \text {; treatment satisfaction } \\
\text { correlated with nail condition; } \\
\text { males expressed more } \\
\text { satisfaction with treatment } \\
\text { than females }(p<0.01)\end{array}$ & $\begin{array}{l}\text { Physician assessment } \\
\text { showed greater } \\
\text { improvement in the } \\
\text { oral therapy group } \\
(p=0.001)\end{array}$ & $\begin{array}{l}9 \text { months, } \\
\text { number lost } \\
\text { to follow-up } \\
\text { NR }\end{array}$ & $\begin{array}{l}\text { No control, no } \\
\text { randomization, } \\
\text { no } \\
\text { standardization } \\
\text { of treatment, no } \\
\text { mycological } \\
\text { confirmation of } \\
\text { diagnosis, } \\
\text { questionable } \\
\text { validity of PRO } \\
\text { instrument }\end{array}$ & 4 \\
\hline $\begin{array}{l}\text { Oral, } \\
\text { Stier et al. } \\
{[28]}\end{array}$ & $\begin{array}{l}\text { Case } \\
\text { series } \\
(n=124)\end{array}$ & $\begin{array}{l}\text { Initial oral } \\
\text { therapy }(n=46) \\
\text { vs. initial } \\
\text { nonoral therapy } \\
(n=78)\end{array}$ & $\begin{array}{l}\text { Toenail Symptom } \\
\text { Index: modified } \\
\text { from Lubeck et al. } \\
{[18-19] \text { (validated, }} \\
\text { OM-specific) }\end{array}$ & $\begin{array}{l}\text { Oral therapy group had greater } \\
\text { QOL effectiveness as measured } \\
\text { by the Toenail Symptom Index } \\
\text { ( } 94 \text { vs. } 49 \% \text { ); an additional } \\
\text { USD } 304-491 \text { was required for } \\
\text { each case improved with oral } \\
\text { medication over } 9 \text { months } \\
\text { though extrapolation showed } \\
\text { cost equivalence after } 17-20 \\
\text { months }\end{array}$ & $\begin{array}{l}\text { Physician global } \\
\text { improvement rating } \\
\text { showed greater } \\
\text { improvement in oral } \\
\text { therapy group }\end{array}$ & $\begin{array}{l}9 \text { months, } \\
\text { number lost } \\
\text { to follow-up } \\
\text { NR }\end{array}$ & $\begin{array}{l}\text { No control, no } \\
\text { randomization, } \\
\text { no } \\
\text { standardization } \\
\text { of treatment, no } \\
\text { mycological } \\
\text { confirmation of } \\
\text { diagnosis }\end{array}$ & 4 \\
\hline
\end{tabular}


Table 2 (continued)

\begin{tabular}{|c|c|c|c|c|c|c|c|c|}
\hline $\begin{array}{l}\text { Treatment } \\
\text { route, } \\
\text { source }\end{array}$ & $\begin{array}{l}\text { Study } \\
\text { design }\end{array}$ & Comparison & PRO instrument & PRO outcomes & Clinical outcomes & Follow-up & Limitations & QOE \\
\hline $\begin{array}{l}\text { Oral, } \\
\text { Firooz et al. } \\
{[29]}\end{array}$ & $\begin{array}{l}\text { Case } \\
\text { series } \\
(n=20)\end{array}$ & $\begin{array}{l}\text { Pulse-dose oral } \\
\text { itraconazole } \\
\text { ( } 200 \text { mg twice } \\
\text { daily for } 1 \text { week } \\
\text { every } 4 \text { weeks } \\
\text { for } 12 \text { weeks); } \\
\text { no control }\end{array}$ & $\begin{array}{l}13 \text { questions from } \\
\text { the instrument by } \\
\text { Drake et al. [12], the } \\
\text { International OM } \\
\text { Questionnaire } \\
\text { (questionable } \\
\text { validity, OM- } \\
\text { specific) }\end{array}$ & $\begin{array}{l}\text { After treatment, patients' } \\
\text { mean QOL scores were } \\
\text { improved }(p=0.009)\end{array}$ & $\begin{array}{l}16 / 20(80 \%) \\
\text { mycologically cured, } \\
9 / 20(45 \%) \text { clinically } \\
\text { cured, } 5 / 20(25 \%) \\
\text { improved, } 6 / 20(30 \%) \\
\text { did not improve }\end{array}$ & $\begin{array}{l}48 \text { weeks, } \\
\text { number lost } \\
\text { to follow-up } \\
\text { NR (but } \\
\text { appears to } \\
\text { be } 0 / 20 \text { ) }\end{array}$ & $\begin{array}{l}\text { Small sample } \\
\text { size, no control, } \\
\text { questionable } \\
\text { validity of PRO } \\
\text { instrument }\end{array}$ & 4 \\
\hline $\begin{array}{l}\text { Oral, } \\
\text { Warshaw et } \\
\text { al. [30] }\end{array}$ & $\begin{array}{l}\text { Cross- } \\
\text { sectional } \\
\text { study } \\
(n=142)\end{array}$ & $\begin{array}{l}\text { Pulse-dose oral } \\
\text { itraconazole } \\
\text { ( } 200 \text { mg twice } \\
\text { daily for } 1 \text { week } \\
\text { every } 4 \text { weeks } \\
\text { for at least } 12 \\
\text { weeks) }(n=48) \\
\text { vs. continuous } \\
\text { oral terbinafine } \\
\text { ( } 250 \text { mg daily } \\
\text { for at least } 12 \\
\text { weeks) }(n=94)\end{array}$ & $\begin{array}{l}\text { Morisky Compliance } \\
\text { Scale (validated), } \\
\text { OnyCOE-t }{ }^{\mathrm{TM}} \\
\text { Satisfaction Scale } \\
\text { (validated, OM- } \\
\text { specific), ease and } \\
\text { convenience scale } \\
\text { (non-validated) }\end{array}$ & $\begin{array}{l}\text { Patients taking terbinafine } \\
\text { reported significantly } \\
\text { increased ease and } \\
\text { convenience }(p=0.008) \text { and } \\
\text { significantly greater overall } \\
\text { satisfaction }(p=0.003) ; \text { no } \\
\text { difference in compliance }\end{array}$ & $\begin{array}{l}\text { NR (cure rates NR for } \\
2 \text { groups) }\end{array}$ & N/A & $\begin{array}{l}\text { Recall bias, no } \\
\text { randomization, } \\
\text { not blinded }\end{array}$ & 4 \\
\hline $\begin{array}{l}\text { Oral, } \\
\text { Potter et al. } \\
{[31]}\end{array}$ & $\begin{array}{l}\text { Open- } \\
\text { label, } \\
\text { RCT } \\
(n=504)\end{array}$ & $\begin{array}{l}\text { Terbinafine } \\
(250 \text { mg daily } \\
\text { for } 12 \text { weeks) } \\
\text { and } \\
\text { debridement of } \\
\text { target toenail } \\
\text { (at } 0,6,12 \text {, and } \\
24 \text { weeks) ( } n= \\
246) \text { vs. } \\
\text { terbinafine only } \\
(n=258)\end{array}$ & $\begin{array}{l}\text { OnyCOE-t }{ }^{\mathrm{TM}} \\
\text { (validated, OM- } \\
\text { specific) }\end{array}$ & $\begin{array}{l}\text { Terbinafine with adjunct } \\
\text { debridement decreases } \\
\text { symptom frequency }(p= \\
0.0395) \text {, especially thickening/ } \\
\text { swelling and discoloration, and } \\
\text { improves overall treatment } \\
\text { satisfaction ( } p=0.0077) \text { in } \\
\text { patients with toenail OM, } \\
\text { though the effect is greatest at } \\
\text { the end of the terbinafine } \\
\text { course }\end{array}$ & NR & $\begin{array}{l}48 \text { weeks, } \\
\text { number lost } \\
\text { to follow-up } \\
\text { NR }\end{array}$ & $\begin{array}{l}\text { Clinical } \\
\text { assessments } \\
\text { were confined } \\
\text { to } 1 \text { nail vs. } \\
\text { PRO measures } \\
\text { disease on all } \\
\text { nails, differences } \\
\text { were minimal as } \\
\text { both groups } \\
\text { received } \\
\text { treatment }\end{array}$ & 1 \\
\hline $\begin{array}{l}\text { Topical, } \\
\text { Malay et al. } \\
{[32]}\end{array}$ & $\begin{array}{l}\text { RCT } \\
(n=55)\end{array}$ & $\begin{array}{l}\text { Debridement } \\
\text { (every } 3 \text { months } \\
\text { for } 9-12 \\
\text { months) and } \\
\text { topical } \\
\text { ciclopirox } 8 \% \\
\text { daily }(n=28) \\
\text { vs. debridement } \\
\text { only }(n=27)\end{array}$ & $\begin{array}{l}\text { Bristol Foot Score } \\
\text { (validated, podiatry- } \\
\text { specific) }\end{array}$ & $\begin{array}{l}\text { Both groups had improved } \\
\text { QOL scores }(p<0.001) \text {, but } \\
\text { adjunct ciclopirox improved } \\
\text { QOL more than debridement } \\
\text { alone }(p=0.002)\end{array}$ & $\begin{array}{l}\text { Debridement alone } \\
\text { did not result in a } \\
\text { single case of } \\
\text { mycological cure } \\
\text { whereas with the } \\
\text { addition of ciclopirox, } \\
\text { mycological cure was } \\
76.7 \%\end{array}$ & $\begin{array}{l}\text { Median } 10.5 \\
\text { months, } \\
2 / 55(4 \%) \\
\text { lost to } \\
\text { follow-up }\end{array}$ & $\begin{array}{l}\text { Participants not } \\
\text { blinded, lab } \\
\text { diagnostics was } \\
\text { only performed } \\
\text { on one toenail }\end{array}$ & 1 \\
\hline $\begin{array}{l}\text { Topical, } \\
\text { Tosti and } \\
\text { Elewski [33] }\end{array}$ & $\begin{array}{l}\text { Double- } \\
\text { blind, } \\
\text { RCT } \\
(n= \\
1,655)\end{array}$ & $\begin{array}{l}\text { Efinaconazole, } \\
10 \% \text {, daily for } \\
48 \text { weeks }(n= \\
1,236) \text { vs. } \\
\text { vehicle }(n= \\
415)\end{array}$ & $\begin{array}{l}\text { OnyCOE-t }{ }^{\mathrm{TM}} \\
\text { (validated, OM- } \\
\text { specific) }\end{array}$ & $\begin{array}{l}\text { Efinaconazole group had } \\
\text { significantly improved mean } \\
\text { QOL scores }(p \leq 0.002) \text {, } \\
\text { significantly higher treatment } \\
\text { satisfaction scores }(p<0.001) \text {, } \\
\text { significantly greater mean } \\
\text { change in all domains ( } p \leq \\
0.002) \text {; there was a significant } \\
\text { correlation between change in } \\
\text { percent affected nail and } \\
\text { change in mean domain scores }\end{array}$ & $\begin{array}{l}\text { Complete cure rate } \\
\text { NR; } 47 \% \text { of patients } \\
\text { treated with } \\
\text { efinaconazole were } \\
\text { considered clinically } \\
\text { improved ( } \leq 10 \% \text { nail } \\
\text { involvement) }\end{array}$ & $\begin{array}{l}52 \text { weeks, } \\
4 / 1,655 \\
(0.2 \%) \text { lost } \\
\text { to follow-up }\end{array}$ & $\begin{array}{l}\text { Time period } \\
\text { may be too } \\
\text { brief, lack of } \\
\text { generalizability, } \\
\text { clinical } \\
\text { assessments } \\
\text { performed on } \\
\text { one toenail but } \\
\text { all nails affect } \\
\text { QOL assessment }\end{array}$ & 1 \\
\hline $\begin{array}{l}\text { Topical, } \\
\text { Snell et al. } \\
{[34]}\end{array}$ & $\begin{array}{l}\text { Case } \\
\text { series } \\
(n=20)\end{array}$ & $\begin{array}{l}\text { Vicks VapoRub, } \\
\text { unclear } \\
\text { treatment } \\
\text { course; no } \\
\text { control }\end{array}$ & $\begin{array}{l}\text { OnyCOE-t }{ }^{\mathrm{TM}} \\
\text { (validated, OM- } \\
\text { specific) }\end{array}$ & $\begin{array}{l}\text { After } 24 \text { weeks, fewer patients } \\
\text { reported embarrassment } \\
(15 / 20,75 \% \text { to } 11 / 18,61 \%) \text { and } \\
\text { pain or discomfort }(11 / 20,53 \% \\
\text { to } 7 / 18,39 \%) \text {, and } 17 / 18(94 \%) \\
\text { of patients reported treatment } \\
\text { satisfaction }\end{array}$ & $\begin{array}{l}\text { After } 24 \text { weeks, } 15 / 18 \\
(83 \%) \text { had } \\
\text { improvement of } \\
\text { affected nails, } 2 / 18 \\
(11 \%) \text { had complete } \\
\text { clearance }\end{array}$ & $\begin{array}{l}48 \text { weeks, } \\
5 / 20(20 \%) \\
\text { lost to } \\
\text { follow-up }\end{array}$ & $\begin{array}{l}\text { Small sample } \\
\text { size, no control, } \\
\text { no blinding, } \\
\text { single site, } \\
\text { compliance } \\
\text { problem, poor } \\
\text { follow-up }\end{array}$ & 4 \\
\hline $\begin{array}{l}\text { Laser, } \\
\text { Ortiz et al. } \\
{[35]}\end{array}$ & $\begin{array}{l}\text { RCT } \\
(n=10)\end{array}$ & $\begin{array}{l}1,320-\mathrm{nm} \\
\text { Nd:YAG laser, } \\
4 \text { treatments } \\
\text { over } 24 \text { weeks } \\
\text { vs. cryogen } \\
\text { spray sham }\end{array}$ & $\begin{array}{l}\text { Nail QOL } \\
\text { Questionnaire } \\
\text { (unclear if validated } \\
\text { as specific } \\
\text { questionnaire is not } \\
\text { cited, OM-specific) }\end{array}$ & $\begin{array}{l}\text { Patients felt less self-conscious } \\
\text { and embarrassed about the } \\
\text { treated toe, but there was no } \\
\text { significant difference from the } \\
\text { control toe }\end{array}$ & $\begin{array}{l}\text { No significant } \\
\text { improvement was } \\
\text { observed in the treated } \\
\text { versus untreated nail }\end{array}$ & NR & $\begin{array}{l}\text { Small sample } \\
\text { size, } \\
\text { confounding } \\
\text { from topical } \\
\text { antifungal and } \\
\text { sham treatment }\end{array}$ & 2 \\
\hline
\end{tabular}

NR, not reported; OM, onychomycosis; PRO, patient-reported outcomes; QOE, quality of evidence; QOL, quality of life; RCT, randomized controlled trial; Nd:YAG, neodymium:yttrium aluminum garnet. 
Table 3. Psychometric properties for evaluating PRO instruments

\begin{tabular}{lll}
\hline Psychometric property & Definition & Quantitative evaluation \\
\hline Variability & $\begin{array}{l}\text { Degree to which the full range of item responses and scale } \\
\text { scores are reported }\end{array}$ & Cronbach a coefficient $>0.70$ \\
\hline $\begin{array}{l}\text { Reliability } \\
\text { Internal consistency } \\
\text { Test-retest }\end{array}$ & $\begin{array}{l}\text { Reproducibility across items } \\
\text { Reproducibility across time }\end{array}$ & $>0.75$ \\
\hline $\begin{array}{l}\text { Validity } \\
\text { Construct } \\
\text { Discriminant } \\
\text { Convergent }\end{array}$ & How well a test measures a variable & \\
\hline \begin{tabular}{l} 
Responsiveness \\
\hline
\end{tabular} & Whether constructs without a relationship are unrelated & \\
\hline
\end{tabular}

in addition to the possibility of an overly sensitive response when no clinical change has occurred.

Drake et al. [12] introduced the International Onychomycosis Questionnaire, which has been evaluated in multiple languages and validated in a Polish cross-sectional study for both toenail and fingernail disease [13]. The toenail-specific scale was also validated in a Serbian crosssectional study [14].

The fifth validated PRO instrument is the NailQoL, which consists of the previously validated Skindex-29 (a dermatological QOL measure) and 10 questions from nonvalidated disease-specific questionnaires [17]. Subjects with complete cure of the target toenail and all 10 toenails reported significantly improved PRO scores $(p \leq$ 0.02).

Finally, Warshaw et al. [30] developed a scale assessing treatment ease and convenience for a cross-sectional study, although a comprehensive onychomycosis instrument was not developed. Warshaw's instrument was valid and responsive with acceptable internal consistency but did not evaluate test-retest reliability.

\section{Treatment}

Ten unique studies used PROs to evaluate treatment in onychomycosis patients, none of which included fingernail disease (Table 2). Patient QOL was examined in 6 studies of oral antifungals, 3 studies of topical antifungals, and 1 study of laser therapy. Only 3 studies used a validated, onychomycosis-specific PRO instrument, the OnyCOE-t ${ }^{\mathrm{TM}}[31,33,34]$. Four studies used instruments derived from previously validated questionnaires [2730], 1 study used a validated instrument for podiatry-re- lated QOL [32], and 2 studies used instruments of unclear origins $[26,35]$. There were 2,224 patients in 4 randomized controlled trials, 1,825 patients in 5 case series, and 142 patients in 1 cross-sectional study.

\section{Impact of Oral Therapies on PRO}

As systemic antifungals are the standard onychomycosis treatment, most QOL data have been collected from onychomycosis patients treated with oral medications. Potter et al. [31] validated the OnyCOE-t ${ }^{\mathrm{TM}}$ in a prospective, randomized, multicenter trial comparing terbinafine alone with the combination of terbinafine and debridement. Both groups experienced QOL improvement. From baseline, debridement significantly improved treatment satisfaction $(p=0.0077)$ and symptom frequency $(p=0.0395)$. Debridement had the most impact in the final week (week 12) of terbinafine treatment; afterwards, the terbinafine-only group had equivocal QOL improvement, suggesting that debridement only had a short-term effect when terbinafine had yet to adequately penetrate the nail plate. While debridement did not cure onychomycosis, it did improve comfort and appearance, thus enhancing QOL.

Multiple studies have used aspects of Lubeck's questionnaire. Stier et al. $[27,28]$ compared oral antifungals to local therapy in 2 case series. In their cost effectiveness study, QOL was assessed with the Toenail Symptom Index, an instrument using 2 reliable, valid, and responsive scales from Lubeck's questionnaire [10]. Oral medications such as terbinafine, itraconazole, and fluconazole were significantly superior to local treatment such as topical treatments or debridement $(p=0.003)$; patients 
Table 4. Content of Five psychometrically evaluated onychomycosis PRO instruments

\begin{tabular}{|c|c|c|c|c|c|}
\hline & Lubeck et al. $[18,19]$ & $\begin{array}{l}\text { International } \\
\text { Onychomycosis } \\
\text { Questionnaire [12] }\end{array}$ & ODSQ [15] & OnyCOE-t ${ }^{\mathrm{TM}}[16]$ & NailQoL [17] \\
\hline Items, $n$ & 80 & 40 & 61 & 33 & 15 \\
\hline Content & $\begin{array}{l}\text { Generic core + disease- } \\
\text { specific (symptoms, } \\
\text { appearance, function, } \\
\text { stigma, overall problem, } \\
\text { treatment satisfaction) }\end{array}$ & $\begin{array}{l}\text { Generic core + } \\
\text { disease-specific } \\
\text { (social, emotion, } \\
\text { and symptoms) }\end{array}$ & $\begin{array}{l}\text { Generic core + } \\
\text { disease specific } \\
\text { (symptom distress, } \\
\text { function, stigma, and } \\
\text { overall problem) }\end{array}$ & $\begin{array}{l}\text { Disease-specific only } \\
\text { (symptoms, } \\
\text { appearance, physical } \\
\text { activities, overall } \\
\text { problem, stigma, } \\
\text { treatment satisfaction) }\end{array}$ & $\begin{array}{l}\text { Disease-specific } \\
\text { only (symptoms, } \\
\text { function, } \\
\text { emotion) }\end{array}$ \\
\hline Validation study & $\begin{array}{l}\text { Multicenter } \\
\text { observational study of } \\
129 \text { patients }\end{array}$ & $\begin{array}{l}\text { Cross-sectional } \\
\text { multicenter study } \\
\text { of } 284 \text { toenail and } \\
248 \text { fingernail } \\
\text { patients in France, } \\
\text { Italy, Germany, } \\
\text { USA }\end{array}$ & $\begin{array}{l}\text { Prospective double- } \\
\text { blind, multicenter } \\
\text { RCT of } 268 \text { patients } \\
\text { comparing antifungal } \\
\text { vs. placebo }\end{array}$ & $\begin{array}{l}\text { Prospective, } \\
\text { multicenter RCT of } 504 \\
\text { patients comparing oral } \\
\text { terbinafine with adjunct } \\
\text { debridement vs. } \\
\text { terbinafine alone }\end{array}$ & $\begin{array}{l}\text { Prospective, RCT } \\
\text { of } 402 \text { patients } \\
\text { comparing two } \\
\text { terbinafine dosing } \\
\text { regimens }\end{array}$ \\
\hline Diagnosis & Clinical & Clinical & Mycological & Mycological & Mycological \\
\hline Location & Toenail & $\begin{array}{l}\text { Toenail and } \\
\text { fingernail }\end{array}$ & $\begin{array}{l}\text { Toenail and } \\
\text { fingernail }\end{array}$ & Toenail & Toenail \\
\hline Variability & Poor (ceiling effects) & $\begin{array}{l}\text { Good (except for } \\
\text { emotional scale) }\end{array}$ & $\begin{array}{l}\text { Good (except for } \\
\text { fingernail symptoms } \\
\text { scale) }\end{array}$ & NR & NR \\
\hline ICR & $\mathrm{CAC} \geq 0.76$ & $\mathrm{CAC} \geq 0.60$ & $\mathrm{CAC} \geq 0.79$ & $\mathrm{CAC} \geq 0.84$ & $\begin{array}{l}\text { CAC } \geq 0.88 \\
\text { (except function } \\
\text { scale) }\end{array}$ \\
\hline TRR & ICC $\geq 0.52$ & ICC $\geq 0.80$ & $\begin{array}{l}\mathrm{ICC} \geq 0.89 \text { (toe) } \\
\mathrm{PCC}=0.79 \text { (finger) }\end{array}$ & NR & ICC $\geq 0.88$ \\
\hline Validity & $\begin{array}{l}\text { Construct (physical } \\
\text { function with } \\
\text { impairment); } \\
\text { discriminant (gender, } \\
\text { age) }\end{array}$ & $\begin{array}{l}\text { Construct (severity } \\
\text { level); discriminant } \\
\text { (duration, extent, } \\
\text { number of nails } \\
\text { affected) }\end{array}$ & $\begin{array}{l}\text { Construct (health } \\
\text { status levels, low vs. } \\
\text { high impact, } \\
\text { treatment outcomes) }\end{array}$ & $\begin{array}{l}\text { Construct (symptom } \\
\text { frequency with bother- } \\
\text { someness, embarrass- } \\
\text { ment with stigma, } \\
\text { treatment satisfaction } \\
\text { with symptom); } \\
\text { discriminant (gender, } \\
\text { age) }\end{array}$ & $\begin{array}{l}\text { Construct } \\
\text { (severity levels) }\end{array}$ \\
\hline Responsiveness & $\begin{array}{l}40 \text { clinically improved } \\
\text { patients vs. } 34 \text { stable } \\
\text { patients }(p<0.05 \text { for all } \\
\text { disease-specific scales } \\
\text { except stigma) }\end{array}$ & NR & $\begin{array}{l}28 \text { clinically cured vs. } \\
168 \text { clinically } \\
\text { improved vs. } 45 \\
\text { failed patients (QOL } \\
\text { change and clinical } \\
\text { improvement } \\
\text { correlation) }\end{array}$ & $\begin{array}{l}400 \text { clinically improved } \\
\text { vs. } 37 \text { stable patients } \\
\text { (Guyatt statistic } \\
\text { indicates moderate to } \\
\text { high responsiveness; } \\
p<0.02 \text { on all scores } \\
\text { except symptom } \\
\text { bothersomeness) }\end{array}$ & $\begin{array}{l}61 \text { complete cure } \\
\text { of all toenails vs. } \\
231 \text { without } \\
(p \leq 0.01) ; 100 \\
\text { complete cure of } \\
\text { target toenail vs. } \\
191 \text { without } \\
(p \leq 0.02)\end{array}$ \\
\hline
\end{tabular}

NR, not reported; RCT, randomized controlled trial; ICR, internal consistency reliability; CAC, Cronbach a coefficient; TRR, testretest reliability; ICC, intraclass correlation coefficient; PCC, Pearson correlation coefficient. Number of items refers to the number of questions included in the tool. Content examines whether the questionnaire included a generic core asking general health questions and what specific categories were included amongst the disease-specific questions. Validation study describes the study design used to validate the tool. Diagnosis states how onychomycosis was diagnosed in the enrolled participants of the validation study. Location indicates whether the tool was validated in toes, fingers, or both. Psychometric validation variables variability, ICR, TRR, validity, and responsiveness are as described in Table 3. 
treated with oral medications also had greater direct medical expenses with an additional cost of USD 304-491 for each case improved over 9 months, although extrapolation showed eventual cost equivalence [28]. The patient satisfaction study by Stier et al. [27] demonstrated that treatment satisfaction was correlated with nail condition, although the content of the questionnaire was unclear. Warshaw et al. [30] used the treatment satisfaction scale from Lubeck's questionnaire in their study of terbinafine versus pulsed-dose itraconazole in a multicenter, crosssectional study with 142 patients. Subjects reported greater ease and satisfaction with terbinafine $(p=0.008$ and $p=0.003$, respectively).

Using an instrument modified from Drake's international questionnaire, Firooz et al. [29] evaluated the impact of itraconazole pulse therapy on the QOL of 20 toenail onychomycosis patients and reported that mean QOL scores significantly improved with treatment. However, with this incomplete instrument modified from Drake's validated tool, no rationale was provided regarding the items chosen for the questionnaire; thus, the reliability and validity of this instrument are in question.

Finally, the earliest use of QOL metrics in onychomycosis treatment was Pollak and Billstein's evaluation of terbinafine efficacy using a large, multicenter, open-label study of 1,534 toenail onychomycosis patients [26]. Patients reported overall satisfaction with the treatment results and improved symptom severity. The details of the PRO instrument were not included.

\section{Impact of Topical Therapies on PRO}

PRO analyses of topical treatments are limited, likely in part because numerous studies have demonstrated the clinical superiority of oral medications [36, 37]. Additionally, until recently, ciclopirox was the only topical drug approved for onychomycosis treatment in the US [36]. With the approval of efinaconazole and tavaborole in 2014, more QOL studies are warranted given the high cost and nonsuperiority of these medications to oral antifungals [37].

Tosti and Elewski [33] used the OnyCOE-t ${ }^{\mathrm{TM}}$ to evaluate the impact of topical efinaconazole versus vehicle on PROs in a 48-week, randomized, multicenter, double-blind study with 1,655 toenail onychomycosis patients. The efinaconazole group had significantly greater mean QOL scores across all domains $(p \leq 0.002)$ and significantly higher treatment satisfaction scores $(p<0.001)$. The improvement was especially marked for the $47 \%$ of subjects who were considered clinically improved. Notably, even subjects in the efinaconazole group who failed to demonstrate clinical improvement had better scores for symptom frequency, appearance, and overall problem as compared to baseline, suggesting that even the smallest change in nail appearance can improve QOL. The change in percent nail affected correlated with change in mean domain scores.

Patients with HIV are at higher risk for onychomycosis and yet are an understudied group. The OnyCOE-t ${ }^{\mathrm{TM}}$ was also used in a 48-week prospective study of Vicks VapoRub for treating toenail onychomycosis in $20 \mathrm{HIV}$ positive subjects [34]. This treatment is a non-FDA-approved modality containing eucalyptus oil, a natural home remedy for onychomycosis with little-to-no evidence of clinical utility. After 24 weeks of treatment, fewer patients reported embarrassment and pain/discomfort, and $94 \%$ of patients reported treatment satisfaction. Limitations of this study included small sample size, poor follow-up, and poor adherence. Additionally, PRO data were not analyzed for statistical significance.

Malay et al. [32] used the Bristol Foot Score, a validated, podiatry-specific PRO instrument to evaluate QOL in 55 toenail onychomycosis patients who underwent either debridement alone or debridement with adjunct ciclopirox treatment in a multicenter randomized controlled trial. Debridement alone did not result in any mycological cure, while the addition of ciclopirox resulted in a cure rate of $76.7 \%$. Both groups had improved QOL scores $(p<0.001)$, but the addition of a topical lacquer improved QOL more than debridement alone $(p=0.002)$.

\section{Impact of Laser Therapy on PRO}

Given that achieving cure for onychomycosis requires prolonged treatment, investigations have turned towards alternative modalities to improve clinical response. Laser, light, and combination therapy provide modalities that free patients from the burden on daily medication adherence and drug-drug interactions [38]. Although there have been hundreds of recent studies examining lasers to treat onychomycosis, PRO analyses are limited. Ortiz et al. [35] investigated the efficacy of the 1,320-nm neodymium:yttrium aluminum garnet laser in a 24 -week, randomized, placebo-controlled study of 10 subjects with bilateral great toe onychomycosis. Overall, no significant improvement was observed in treated versus untreated nails. A questionnaire was used to evaluate PRO, although its contents were not identified. Patients reported less self-consciousness and embarrassment about treated toenails despite no clinical difference. Limitations of this study included small sample size, concomitant treatment with a topical antifungal, and possible therapeutic effect of the sham treatment.
152

Skin Appendage Disord 2017;3:144-155 DOI: $10.1159 / 000469666$
Wang/Wiznia/Rieder 


\section{Discussion}

Although onychomycosis has traditionally been viewed as a cosmetic problem, PRO analyses have demonstrated the substantial morbidity of this disease $[5,7$, 10, 18-22]. This review identified 5 psychometrically evaluated instruments that can evaluate patient QOL: Lubeck's [10] and Drake's [12] instruments as well as the ODSQ [15], OnyCOE-t ${ }^{\mathrm{TM}}$ [16], and NailQoL [17]. Only the OnyCOE-t $\mathrm{t}^{\mathrm{TM}}$ has been used in its entirety $[31,33,34]$. In practice, patient responses in these QOL instruments have paralleled clinical change, which lends to their credibility. Additionally, PRO instruments allow practitioners to codify and translate subjective patient experiences into quantitative data.

While onychomycosis is not life-threatening, in studies of the effect of onychomycosis on QOL, $92 \%$ of patients reported negative psychosocial or physical impairments [22], and $70 \%$ of patients considered onychomycosis to be at least a moderate problem [23]. Compared to controls, onychomycosis patients had significantly poorer QOL in general health perception, bodily pain, mental health, social functioning, health concern $[18,19]$, physical appearance [13, 14, 17-19, 25], and physical functioning, such as limitations with standing and working with one's fingers [12, 14, 18-21, 23]. Onychomycosis patients were more self-conscious $[17-19,22,25]$ and embarrassed [12, 13, 17-19, 21, 23], feared spread of transmission to other nails or other people $[12-14,22,25]$, and experienced pain $[12,13,17-23]$. In using the validated, dermatology-specific instrument Skindex-29, onychomycosis QOL impairment was worse than other nail disorders [7], comparable to nonmelanoma skin cancer and benign growths [17], and better than eczema and body psoriasis [17]. This morbidity is not always readily evident. Pain associated with onychomycosis is often underestimated by physicians [12], and although clinicians may not appreciate any clinical change after treatment, patients report improved QOL [33].

To assess patient QOL, 5 PRO instruments have been validated for onychomycosis. Drake's questionnaire is the only instrument validated in multiple languages but also the only one without data on responsiveness, thus limiting its use in monitoring disease activity. Drake's questionnaire and the ODSQ are the only instruments validated for fingernail disease, although the ODSQ does not explicitly list fingernail-specific items. The validation studies for the ODSQ, OnyCOE- $\mathrm{t}^{\mathrm{TM}}$, and NailQoL established diagnosis by mycological criteria; the validity of Lubeck's and Drake's instruments may be questionable as

Onycho PRO patients could have had nonfungal nail disease. Though earlier validated questionnaires included general health measures, the OnyCOE-t ${ }^{\mathrm{TM}}$ and NailQoL assess only onychomycosis-specific items. A generic core allows comparisons across interventions and diseases as it uses previously validated measures to evaluate parameters of general health distress, psychosocial well-being, and physical functioning $[7,17,24]$. However, this core also has low sensitivity to group differences and clinical changes [10, $12,15,17-19]$. It also lengthens questionnaires, making clinical implementation less feasible [9], which may explain why Lubeck's and Drake's questionnaires have not been utilized in their entirety though selections from each have been appropriated [27-30]. To date, only the OnyCOE- $\mathrm{t}^{\mathrm{TM}}$ has been used in full $[31,33,34]$. The OnyCOE-t tM's $^{\mathrm{TM}}$ popularity may be secondary to its shorter length and defined MCID, which allows clinicians to correlate PRO data with clinical change.

The majority of PRO data for treatment assessment parallel clinical data: oral antifungals have higher cure rates and also larger QOL changes when compared to topicals [26-28]; terbinafine is superior for clinical improvement and treatment satisfaction when compared to pulsed-dose itraconazole [30]. However, with new topical antifungal medications and increasing interest in laser and light therapy, PROs are important to understand patient priorities. In a 1998 study, although $79 \%$ of patients asked about treatment for onychomycosis, $26 \%$ reported that their providers discouraged medication use [19]. Even if insurance would not cover the cost, $97 \%$ of patients were willing to pay for medications with an $80 \%$ cure rate; $57 \%$ were still willing to pay even if the cure rate dropped to $35 \%$ [23]. This is particularly relevant given the recent introduction of expensive topical therapies for onychomycosis. Efinaconazole has been reported to result in improvement in only $42.6 \%$ of patients, and yet has been reported to improve QOL domains even in patients without appreciable clinical improvement, which further emphasizes that patient QOL cannot be deduced from clinical exam alone [33]. PRO instruments are essential for optimizing treatment to maximize compliance, treatment satisfaction, and QOL.

It should be noted that some studies are limited by their study design. For example, Ortiz et al. [35] showed that patients undergoing laser treatment had QOL improvement without significant clinical change, suggesting that laser therapy can be worthwhile for onychomycosis patients; however, the validity of the conclusion is limited by a small sample size and concurrent antifungal use. Additionally, the effect of disease recurrence on pa-

Skin Appendage Disord 2017;3:144-155 DOI: $10.1159 / 000469666$ 
tient QOL and willingness to pay was not evaluated. Given such limitations, study results should be extrapolated with caution, particularly when patients may face exorbitant expenses for a therapy that may not be clinically effective or offer long-term, sustained results.

It is worth noting that the reviewed literature does not address the differences between subjects presenting with a specific complaint of onychomycosis versus those in which the disease is an incidental, and sometimes unconcerning, finding. Research subjects and patients seeing a specialist for onychomycosis are self-selecting and may disproportionately reflect QOL impairment. Given that, it is difficult to quantify the extent of QOL impairment, or perhaps even to firmly establish that onychomycosis is the cause of QOL impairment without comparison to a non-self-selecting control group with incidental onychomycosis. It is possible that QOL impairment may not be a reflection of onychomycosis per se, but more a marker of a threshold of QOL impairment necessary for patients to present to a dermatologist.

Future research should include psychometric evaluation of these instruments in special populations, fingernail disease, and between patients self-selecting for onychomycosis and controls with onychomycosis as incidental findings. For example, HIV and diabetes mellitus are risk factors for onychomycosis and may modify disease type and course, but most PRO trials exclude patients with these comorbidities. Additionally, neither the OnyCOE- $\mathrm{t}^{\mathrm{TM}}$ nor the NailQoL instrument is validated for fingernail disease despite the fact that fingernail involvement has been shown to increase patient disease burden $[17,23]$.

\section{Conclusion}

As medical practice increasingly emphasizes patientcentered care and shared decision-making, QOL assessments are becoming increasingly important. Through PRO instruments, it is apparent that onychomycosis is not only a cosmetic hindrance but also a burdensome medical disorder that patients want and are willing to treat, at times even for limited or no clinically apparent benefit. Five PRO questionnaires have been validated for onychomycosis, and they have been infrequently used in clinical studies examining treatment efficacy. Future research should utilize these instruments to generate further insight into patients' assessment of their health status and of new therapies.

\section{Disclosure Statement}

The authors have no conflicts of interest to declare. There were no funding sources for this project.

\section{References}

1 Gupta AK, Jain HC, Lynde CW, MacDonald P, Cooper EA, Summerbell RC: Prevalence and epidemiology of onychomycosis in patients visiting physicians' offices: a multicenter Canadian survey of 15,000 patients. J Am Acad Dermatol 2000;43:244-248.

2 Rosen T, Friedlander SF, Kircik L, Zirwas MJ, Stein Gold L, Bhatia N, et al: Onychomycosis: epidemiology, diagnosis, and treatment in a changing landscape. J Drugs Dermatol 2015; 14:223-233.

3 Rosenbach M, Schneider J: The Burden of Onychomycosis in the Medicare Population. Health Economic Research Inc, Sandoz Pharmaceuticals Corporation, 1990.

4 Scher RK: Onychomycosis: a significant medical disorder. J Am Acad Dermatol 1996; 35:S2-S5.

5 Elewski BE: Onychomycosis. Treatment, quality of life, and economic issues. Am J Clin Dermatol 1999;1:19-26.

6 Scher RK: Onychomycosis is more than a cosmetic problem. Br J Dermatol 1994;130(suppl 43):15.
7 Tabolli S, Alessandroni L, Gaido J, Sampogna F, Di Pietro C, Abeni D: Health-related quality of life and nail disorders. Acta Derm Venereol 2007;87:255-259.

8 Van Cranenburgh O, Prinsen C, Sprangers M, Spuls PI, de Korte J: Health-related quality-of-life assessment in dermatologic practice: relevance and application. Dermatol Clin 2012;30:323-332.

9 Shaw JW, Joish VN, Coons SJ: Onychomycosis. Pharmacoeconomics 2002;20:23-36.

10 Lubeck D, Gause D, Schein J, Prebil L, Potter L: A health-related quality of life measure for use in patients with onychomycosis: a validation study. Qual Life Res 1999;8:121-129.

11 Lubeck DP, Schein JR, Gause D, Prebil LA, Potter LP: Health-related quality of life in patients with toenail onychomycosis: data from a 9-month observational study. J Clin Outcomes Manag 1999;6:37-46.

12 Drake LA, Patrick DL, Fleckman P, André J, Baran R, Haneke E, et al: The impact of onychomycosis on quality of life: development of an international onychomycosis-specific questionnaire to measure patient quality of life. J Am Acad Dermatol 1999;41:189-196.
13 Szepietowski J, Reich A, Pacan P, Garlowska E, Baran E: Evaluation of quality of life in patients with toenail onychomycosis by Polish version of an international onychomycosisspecific questionnaire. J Eur Acad Dermatol Venereol 2007;21:491-496.

14 Milobratović D, Janković S, Vukičević J, Marinković J, Janković J, Railić Z: Quality of life in patients with toenail onychomycosis. Mycoses 2013;56:543-551.

15 Turner RR, Testa MA: Measuring the impact of onychomycosis on patient quality of life. Qual Life Res 2000;9:39-53.

16 Potter LP, Mathias SD, Raut M, Kianifard F, Tavakkol A: The OnyCOE- ${ }^{\mathrm{TM}}$ questionnaire: responsiveness and clinical meaningfulness of a patient-reported outcomes questionnaire for toenail onychomycosis. Health Qual Life Outcomes 2006;4:1.

17 Warshaw EM, Foster JK, Cham PM, Grill JP, Chen SC: NailQoL: a quality-of-life instrument for onychomycosis. Int J Dermatol 2007;46:1279-1286. 
18 Lubeck D, Patrick D, McNulty P, Fifer S, Birnbaum J: Quality of life of persons with onychomycosis. Qual Life Res 1993;2:341-348.

19 Lubeck DP: Measuring health-related quality of life in onychomycosis. J Am Acad Dermatol 1998;38:S64-S68.

20 Whittam L, Hay R: The impact of onychomycosis on quality of life. Clin Exp Dermatol 1997;22:87-89.

21 Schein J, Gause D, Stier D, Lubeck D, Bates M, Fisk R: Onychomycosis. Baseline results of an observational study. J Am Podiatr Med Assoc 1997;87:512-519.

22 Elewski BE: The effect of toenail onychomycosis on patient quality of life. Int J Dermatol 1997;36:754-756.

23 Drake LA, Scher RK, Smith EB, Faich GA, Smith SL, Hong JJ, et al: Effect of onychomycosis on quality of life. J Am Acad Dermatol 1998;38:702-704.

24 Bunyaratavej S, Pattanaprichakul P, Leeyaphan C, Chayangsu O, Bunyaratavej S, Kulthanan K: Onychomycosis: a study of selfrecognition by patients and quality of life. Indian J Dermatol Venereol Leprol 2015;81: 270-274.

25 Szepietowski JC, Reich A: Stigmatisation in onychomycosis patients: a population-based study. Mycoses 2009;52:343-349.

26 Pollak R, Billstein SA: Efficacy of terbinafine for toenail onychomycosis: a multicenter trial of various treatment durations. J Am Podiatr Med Assoc 2001;91:127-131.
27 Stier DM, Gause D, Joseph WS, Schein JR, Broering JM, Warolin KL, et al: Patient satisfaction with oral versus nonoral therapeutic approaches in onychomycosis. J Am Podiatr Med Assoc 2001;91:521-527.

28 Stier DM, Henke C, Schein J, Doyle J, Schonfeld $\mathrm{WH}$, Broering J: Cost of treatment for onychomycosis. Pharmacoeconomics 2001; 19:267-279.

29 Firooz A, Khamesipour A, Dowlati Y: Itraconazole pulse therapy improves the quality of life of patients with toenail onychomycosis. J Dermatolog Treat 2003;14:95-98.

30 Warshaw EM, Bowman T, Bodman MA, Kim JJ, Silva S, Mathias SD: Satisfaction with onychomycosis treatment: pulse versus continuous dosing. J Am Podiatr Med Assoc 2003;93: 373-379.

31 Potter LP, Mathias SD, Raut M, Kianifard F, Landsman A, Tavakkol A: The impact of aggressive debridement used as an adjunct therapy with terbinafine on perceptions of patients undergoing treatment for toenail onychomycosis. J Dermatolog Treat 2007; 18: 46-52.
32 Malay DS, Yi S, Borowsky P, Downey MS, Mlodzienski AJ: Efficacy of debridement alone versus debridement combined with topical antifungal nail lacquer for the treatment of pedal onychomycosis: a randomized, controlled trial. J Foot Ankle Res 2009;48: 294-308.

33 Tosti A, Elewski BE: Treatment of onychomycosis with efinaconazole $10 \%$ topical solution and quality of life. J Clin Aesthet Dermatol 2014;7:25-30.

34 Snell M, Klebert M, Önen NF, Hubert S: A novel treatment for onychomycosis in people living with HIV infection: Vicks VapoRub ${ }^{\mathrm{TM}}$ is effective and safe. J Assoc Nurses AIDS Care 2016;27:109-113.

35 Ortiz AE, Truong S, Serowka K, Kelly KM: A 1,320-nm Nd:YAG laser for improving the appearance of onychomycosis. Dermatol Surg 2014;40:1356-1360.

36 Gupta AK, Daigle D, Foley KA: Topical therapy for toenail onychomycosis: an evidencebased review. Am J Clin Dermatol 2014;15: 489-502.

37 Gupta AK, Daigle D, Foley KA: Network meta-analysis of onychomycosis treatments. Skin Appendage Disord 2015;1:74-81.

38 Wiznia LE, Quatrano NA, Mu EW, Rieder EA: A clinical review of laser and light therapy for nail psoriasis and onychomycosis. Dermatol Surg 2017;43:161-172. 